UVX 2008 (2009) 173-176

(C) EDP Sciences, 2009

DOI: $10.1051 / \mathrm{uvx} / 2009028$

\title{
Study on the transfer induced by laser of organic conducting thin films
}

\author{
L. Rapp, C. Cibert, A.P. Alloncle and P. Delaporte \\ Lasers, Plasmas et Photonic Processes Laboratory (LP3), UMR 6182 CNRS University \\ of Méditerranée, C. 917, 13288 Marseille Cedex 9, France
}

\begin{abstract}
The Laser-Induced Forward Transfer (LIFT) [1] technique has been performed on thin layers of organic conducting material for applications in plastic micro-electronics. This simple, single step, direct printing technique enables to make surface micro patterning or localized deposition of material. This process is a promising alternative for manufacture of organic electronic components on flexible supports when usual techniques, such like ink jet printing, cannot be considered. For instance, when the organic material has no solubility properties or when complex architectures are needed. It can be applied to sensitive materials without altering their properties $[2,3]$. This opens the way to alternative manufacturing processes for the Organic Field-Effect Transistor (OFET) technology. The ejected material is highly forward directed, with an angular divergence on the order of $3^{\circ}$ while propagating over a distance of $2 \mathrm{~mm}$. The high directivity of the ejection with picosecond pulses duration shows that complex micro-structures with a high spatial resolution can be performed using the no contact LIFT technique. The influence of the distance between the donor and acceptor substrates on process reliability is also discussed. Moreover no limitation is expected to miniaturization, which is a necessary requirement.
\end{abstract}

\section{INTRODUCTION}

New conductive polymers used in plastic micro-electronics are regularly synthesized, but numbers of them do not have the solubility properties which would allow their deposition using ink-jet techniques. LIFT process gives the possibility to produce electronic components with non-soluble organic materials. In this work, our objective is to study the LIFT process of a conducting polymer, the poly $(3,4-$ ethylenedioxythiophene)-poly(styrenesulfonate) (PEDOT:PSS) [4]. Its electrical properties and its high stability in time make it a good candidate for the fabrication of the source and drain of an organic thin film transistor (OTFT). The LIFT transferred PEDOT:PSS structures must be spatially well defined in order to get a channel length as short as possible and the homogeneity and electrical properties of the material must be kept.

\section{EXPERIMENTAL PROCESS}

In order to understand the basic mechanism of the transfer, the dynamic of the ejection of this organic material has been investigated by time resolved shadowgraphic imaging from the laser irradiation pulse to $1.5 \mu \mathrm{s}$, with $20 \mathrm{~ns}$ time resolution $[5,6]$. The ejection has been carried out using 50 picoseconds laser pulse with a Nd:YAG (Leopard S10/20 Continuum) at $355 \mathrm{~nm}$ (3rd harmonic) under atmospheric conditions. At this wavelength, the film of PEDOT:PSS is partially transparent to this incident radiation and it is estimated that only $35 \%$ of the incident energy is trapped in the layer. Previous studies have shown that it is preferable to use an irradiation wavelength which is absorbed in the first nanometers of the layer in order to confine damages resulting of the thermal and photochemical effects during the interaction [7]. Gold has been used because of its mechanical properties and its short absorption length for UV radiation (gold: $l_{a b s}=15 \mathrm{~nm} @ 355 \mathrm{~nm}$ )[8]. However it can bring complications in contaminating the deposited material with debris resulting from its ablation. We have determined the 
velocity of the ejection of the transferred material and studied the influence of a sacrificial gold Dynamic Release Layer (DRL) using a wide range of fluences.

Gold layers have been deposited by thermal evaporation in high vacuum (BocEdwards Auto306 Vacuum Coating System) on quartz suprasil substrates with various thicknesses $(10,25$ and $50 \mathrm{~nm})$. Thin films of PEDOT:PSS have been spin-coated on transparent substrates of quartz suprasil with and without DRL. The samples have then been annealed at $80^{\circ} \mathrm{C}$ during 20 minutes to evaporate the solvent. The PEDOT:PSS thickness is quite homogeneous and varies from $300 \mathrm{~nm}$ to $320 \mathrm{~nm}$. Samples have been characterized by optical microscopy using a Zeiss Axiotech with a Nomarski objective and by scanning electronic microscopy (SEM) (Jeol JSM-6390). Electrical properties have been characterized using I(V) measurements with a four-point probe Süss Microtec PM5 system.

\section{RESULTS AND DISCUSSION}

\subsection{Shadowgraphic visualisations}

Series of shadowgraphic images of the ejected PEDOT:PSS layer with and without a $25 \mathrm{~nm}$ gold DRL are taken at various delay times after the laser irradiation. In the small volume delimited by the penetration depth of the beam and the irradiated surface, the radiation intensity is very high $\left(\approx 2 \cdot 10^{9}\right.$ to $10 \cdot 10^{9} \mathrm{~W} / \mathrm{cm}^{2}$ ) and the absorbed energy is dissipated on a thermo-mechanical way including formation and propagation of a shock wave, ionisation, vaporization and ablation of the material. The Figure 1

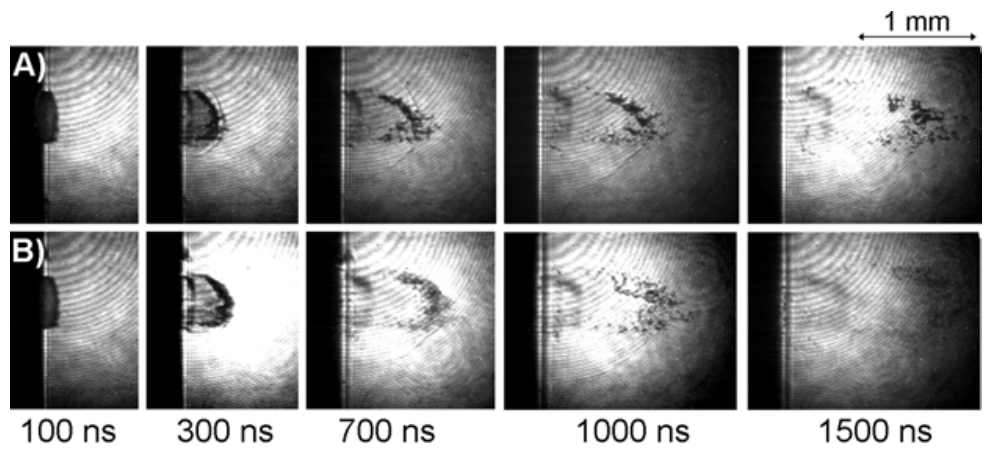

Figure 1. Shadowgraphic images of ejected PEDOT:PSS at various delay times after laser irradiation at $0.6 \mathrm{~J} / \mathrm{cm}^{2}$. A series PEDOT:PSS alone. B series PEDOT:PSS with $25 \mathrm{~nm}$ of gold DRL.

clearly illustrates the ejection of the layers and the propagation of the wave. The absorption of the beam takes places in the gold layer which is vaporized and the polymer is ejected under mechanical stresses. It keeps its cohesion during a short time and becomes quickly fragmented.

The ejected materials are highly forward directed, with an angular divergence on the order of $3^{\circ}$ for PEDOT:PSS and $4^{\circ}$ for PEDOT:PSS with a gold DRL while propagating over a distance of $2 \mathrm{~mm}$. However the materials are propagating in air and some perturbations could induce a loose of cohesion.

\subsection{Comparison on velocities}

The front velocities of the ejected polymer with and without gold inserted are reported in the table 1 .

It is interesting to note the efficiency of the DRL to transfer a higher kinetic energy to the organic layer. The speed of the ejection increases when a gold DRL is inserted. For fluences equal and higher than $0.4 \mathrm{~J} / \mathrm{cm}^{2}\left(0.2 \mathrm{~J} / \mathrm{cm}^{2}\right.$ with DRL), the organic material is propagating faster than the blast wave that could induced serious damages in his cohesion. 
Table 1. Velocities of the ejected conducting polymer with and without gold.

\begin{tabular}{|c|ccccc|}
\hline Fluence $\left(\mathrm{J} / \mathrm{cm}^{2}\right)$ & \multicolumn{2}{|c|}{ Front velocity of PEDOT:PSS } & \multicolumn{2}{c|}{ Front velocity of PEDOT:PSS with $25 \mathrm{~nm}$ of gold } \\
\cline { 2 - 5 } & Ejected material & Wave & Ejected material & Wave \\
\hline 0.1 & $230 \mathrm{~m} / \mathrm{s}$ & $430 \mathrm{~m} / \mathrm{s}$ & $260 \mathrm{~m} / \mathrm{s}$ & $410 \mathrm{~m} / \mathrm{s}$ \\
0.2 & $590 \mathrm{~m} / \mathrm{s}$ & $640 \mathrm{~m} / \mathrm{s}$ & $730 \mathrm{~m} / \mathrm{s}$ & $650 \mathrm{~m} / \mathrm{s}$ \\
0.4 & $900 \mathrm{~m} / \mathrm{s}$ & $820 \mathrm{~m} / \mathrm{s}$ & $1120 \mathrm{~m} / \mathrm{s}$ & $740 \mathrm{~m} / \mathrm{s}$ \\
0.6 & $1140 \mathrm{~m} / \mathrm{s}$ & $970 \mathrm{~m} / \mathrm{s}$ & $1530 \mathrm{~m} / \mathrm{s}$ & $840 \mathrm{~m} / \mathrm{s}$ \\
\hline
\end{tabular}

\subsection{Analyse of the deposits}

\subsubsection{Transfer in contact LIFT technique}

The material transfer in contact LIFT technique is studied in order to determine the optimal conditions of irradiation. The fluence is gradually varied from insufficient conditions to break the donor film to an excessive irradiation. Figure 2(A) displays two deposits obtained in the best condition of transfer i.e. an homogeneous surface and square shape with well-defined edge [7]. They are obtained for a fluence of $0.6 \mathrm{~J} / \mathrm{cm}^{2}$. A little debris appear around the deposits but it can be removed very easily with a soft air blow. For gold layer the best transfer is obtained when the thickness of the polymer film is twice the absorption length of the material (in this case $25 \mathrm{~nm}$ ). Figure 2(B) shows a SEM visualization of a deposit realized in the best condition when a gold DRL of $25 \mathrm{~nm}$ is inserted. The edges are precisely defined and no particles appear all around the deposit, pointing out the benefit of using a thin sacrificial DRL. But X-ray dispersive energy analyses revealed a contamination of the entire surface by microand nanoparticles of gold. A close view to the surface showing a micro-droplet of gold are presented on figure 2(C).

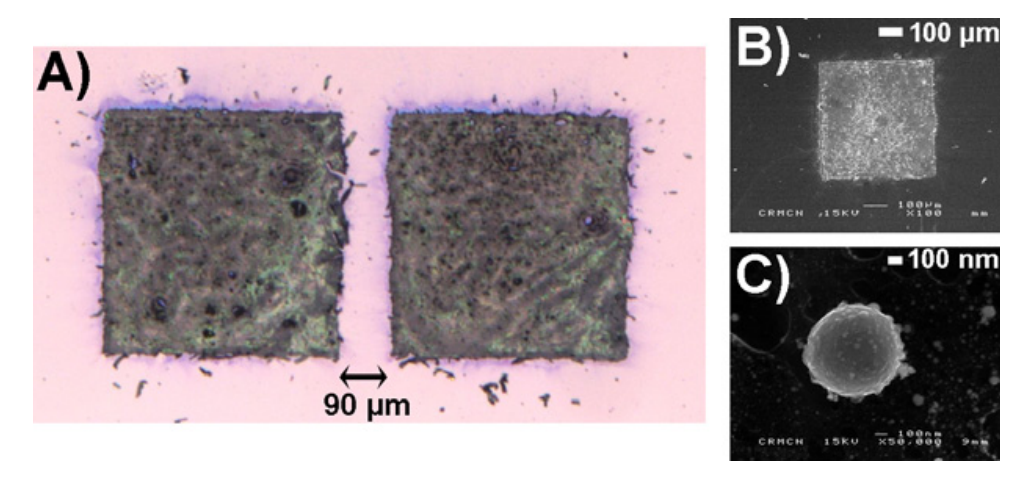

Figure 2. PEDOT:PSS deposit obtained in the best condition by LIFT. Fig. (A). is an optical microscopy image of two deposits. Fig. (B). is a SEM visualization of a deposit transfer with $25 \mathrm{~nm}$ of DRL. Fig. (C). is a close view on the surface of fig. (B).

\subsubsection{Electrical properties}

We have tested the electrical properties of the PEDOT:PSS before and after the LIFT experiments. First we have characterized layers on donor substrates and obtained an average resistivity value of $5 \cdot 10^{-3} \Omega \cdot \mathrm{cm}$. Then lines have been made by LIFT in juxtaposing deposits with different overlapping values and $\mathrm{I}(\mathrm{V})$ measurements have been performed at different distances on these lines. The different measurements for a same distance show a good reproducibility of the characteristics but point out also the difficulty to keep the electrical contact between two juxtaposed spots. The value of the resistance increases with the distance of measurement. From these measurements, an average resistivity of the deposits is estimated to be $5 \cdot 10^{-3} \Omega \mathrm{cm}$ and the associated conductivity is $200 \mathrm{~S} \cdot \mathrm{cm}^{-1}$. This is an 
interesting resistivity value in comparison with the constructor value 0.01 to $0.02 \Omega \cdot \mathrm{cm}$ and printing PEDOT:PSS value $13 \cdot 10^{-3} \Omega \cdot \mathrm{cm}$ [9], moreover the transferred deposits are not altered after the LIFT experiments. We have obtained the same values for the deposits transferred with $25 \mathrm{~nm}$ of gold, so in this case, the gold DRL do not affect the measurement.

\section{CONCLUSION}

We have reported in this paper LIFT experiments on a conducting polymer under different conditions of irradiation. The ejection has been studied by time resolved shadowgraphic imaging with and without gold DRL to obtain a better comprehension of the transfer during the LIFT experiments. The deposits keep a good cohesion during the transfer. We have shown the benefit of using a sacrificial metallic layer and the importance of its thickness but there is a risk of contamination. That is why using an insulator with absorbing properties could be a good idea in order to optimize the process [10]. Moreover experiments using $266 \mathrm{~nm}$ picosecond radiation can be performed with no DRL on PEDOT:PSS to take advantage of its high absorption at this wavelength.

The high directivity of the ejection and the distance of propagation with picosecond pulses show that complex micro-structures with a high spatial resolution can be performed using the no contact LIFT technique. However the maximum distance between the donor and the acceptor substrate to obtain good deposit is very short (only few hundred micrometers).

The transferred film has interesting electrical properties, showing that conducting polymer can be transferred by LIFT process. Therefore no limitation is expected to miniaturization, which is a necessary requirement.

\section{Acknowledgements}

The authors would like to acknowledge scientists from the CINaM (Marseille) and the CMP-GC (Gardanne) for their collaboration within the MICROPOLY framework (CIMPACA project) and the ANR program e-PLAST.

\section{References}

[1] J. Bohandy, B.F. Kim, F.J. Adrian, A.N. Jette, J. Appl. Phys. 63 (4), (1988) 1158.

[2] D. B. Chrisey et al, Chem. Rev. 103, (2003) 553-576

[3] J. M. Fernández-Pradas et al, Thin Solid Films, 453-454, (2004) 27-30

[4] B. Thomas, A.P. Alloncle, P. Delaporte, M. Sentis, S. Sanaur, M. Barret, P. Collot, Appl. Surf. Sci. 254, (2007) 1206.

[5] I. Zergioti et al, Appl. Surf. Sci. 197-198, (2002) 868.

[6] I. Zergioti et al, Appl. Surf. Sci. 247, (2005) 584.

[7] L. Rapp, C. Cibert, A.P. Alloncle, P. Delaporte, Appl. Surf. Sci. 255, (2009) 5439-5443

[8] Michael Bass (Ed.), Handbook of optics, (second edition, 1995).

[9] M. Barret, Impression par jet de matière de transistors organiques sur support souple, Thèse de doctorat de l'Ecole Nationale Supérieure des Mines de Saint-Etienne soutenue le 12.10.07.

[10] R. Fardel, M. Nagel, F. Nüesch, T. Lippert, A. Wokaun, Appl. Surf. Sci. 254, (2007) 1322. 